\title{
Voronoi Drawings of Trees ${ }^{\star}$
}

\author{
Giuseppe Liotta ${ }^{1}$ and Henk Meijer ${ }^{2}$ \\ 1 Dipartimento di Ingegneria Elettronica e dell'Informazione, \\ Università di Perugia, Perugia, Italy. \\ liotta@diei.unipg.it \\ 2 Department of Computing and Information Science, \\ Queen's University, Kingston, Ontario, Canada. \\ henk@cs . queensu.ca
}

\begin{abstract}
This paper investigates the following problem: Given a tree $T$, can we find a set of points in the plane such that the Voronoi diagram of this set of points is a drawing of $T$ ? We study trees that can be drawn as Voronoi diagrams both in the Euclidean and in the Manhattan metric. Characterizations of drawable trees are given and different drawing algorithms that take into account additional geometric constraints are presented.
\end{abstract}

\section{Introduction}

Voronoi diagrams are one of the most studied structures in computational geometry because of their use in key application areas including metrology, astronomy, biology, GIS, computer graphics, and operations research $[1,12]$. Often papers and book chapters that are devoted to Voronoi diagrams simplify the matter adopting the "no-degeneracies assumption", i.e assuming that no four sites defining the diagram are co-circular (see, e.g., [13]). Under this assumption, Voronoi diagrams in the Euclidean metric have all vertices of degree three. However, the increasing demand of robust and reliable computational geometry methodologies in recent years has been leading to re-studying basic geometric problems and structures within more realistic frameworks in which simplifying assumptions that rule out degenerate configurations of the input are avoided.

This paper studies degenerate Voronoi diagrams, i.e. Voronoi diagrams that contain vertices of degree higher than three originated by four or more co-circular points (also called sites in the following). Based on the observation that a typical degeneracy for Voronoi diagrams is the one in which co-circular points give rise to a tree-like structure (see, e.g. [14,12]) we investigate the combinatorial properties of degenerate Voronoi diagrams by asking what type of trees can be the Voronoi diagram of some set of sites in the plane. This question can be naturally turned into the following graph drawing problem: Given a tree $T$, can one represent $T$

\footnotetext{
* Research supported in part by the CNR Project "Geometria Computazionale Robusta con Applicazioni alla Grafica ed al CAD', by the ESPRIT LTR Project 20244 (ALCOM-IT), and by NSERC. The authors wish to thank G. Di Battista for support and useful discussions.
} 
so that the resulting drawing is the Voronoi diagram of some set of sites in the plane?

We recall that the the problem of analyzing the combinatorial properties of a given type of geometric graph has a long tradition in the computational geometry and graph drawing communities [2]. We give a few examples below. Monma and Suri [11] proved that each tree with maximum vertex degree at most five can be the Euclidean minimum spanning tree of some set of vertices in the plane, while no tree having at least one vertex with degree greater than six can be drawn as a minimum spanning tree on the plane. As for trees having maximum degree equal to six, Eades and Whitesides [7] showed that it is NPhard to decide whether such trees can be Euclidean minimum spanning trees on the plane. The problem of representing a tree as a Euclidean minimum spanning tree in the three dimensional space is studied in [8]. Dillencourt has shown that all Delaunay drawable triangulations are 1-tough, and have perfect matchings [5], and that all maximal outerplanar graphs are Delaunay drawable [4]. Dillencourt and Smith [6] show that any triangulation without chords or non facial triangles is Delaunay drawable. The problem of deciding whether a graph can be drawn as rectangle of influence graph and the design of efficient algorithms for computing such a drawing when one exists is investigated in [9]. A survey on the problem of drawing a graph as a given type of geometric graph can be found in [3].

Representing a tree as a Voronoi diagram has many interesting aesthetic features. It captures the natural way of drawing a tree so that groups of adjacent vertices appear close to each other while vertices not incident to a certain edge are drawn far apart from that edge. Also, the shape of the edges varies according to the chosen metric for the Voronoi diagram: in the Euclidean metric the edges follow the straight-line standard, i.e. they are straight lines, while in the Manhattan metric the edges follow the semi-orthogonal standard, i.e. they are polygonal chains of segments with slope $0, \infty$, or \pm 1 . A drawing of a graph that is the Voronoi diagram of a set of sites when distances are measured according to the Euclidean metric (also called $L_{2}$ metric) is a Euclidean Voronoi drawing. Similarly, a Manhattan Voronoi drawing is a drawing of a graph that is the Voronoi diagram of some set of sites in the plane when distances are measured according to the Manhattan metric (also called $L_{1}$ metric). For display purposes, we assume that in a Voronoi drawing all edges have finite length, that is the infinite rays of a Voronoi diagram are replaced by edges of finite length in a Voronoi drawing. A graph that admits a Euclidean (Manhattan) Voronoi drawing is said to be Euclidean (Manhattan) Voronoi drawable. Figure 1 (a) and (b) shows a Euclidean and a Manhattan Voronoi drawing of a tree, respectively. In both figures, the white circles represent the sites whose Voronoi diagram is the drawing of the tree.

It is worth mentioning that the requirement of clustering together adjacent vertices has been already considered in the context of proximity drawings, a family of straight-line drawings of graphs which include Gabriel drawings, relative neighborhood drawings, and $\beta$-drawings among its representatives. In a proximity drawing two vertices are adjacent if some local proximity constraint 


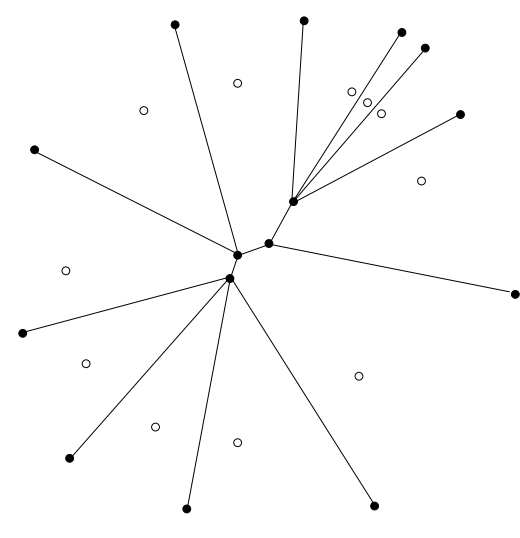

(a)

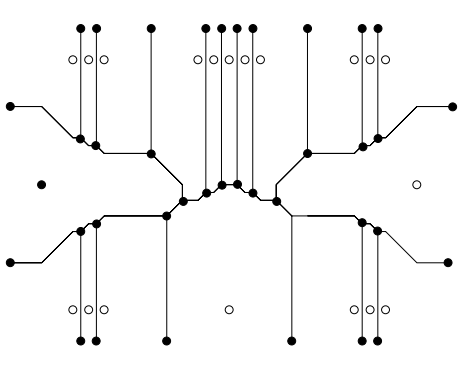

(b)

Fig. 1. (a) Euclidean Voronoi drawing and (b) Manhattan Voronoi drawing of a tree. The white circles are sites, the black circles are vertices of the drawing.

is satisfied; for example, in a Gabriel drawing it is required that the disk having the two vertices as antipodal points does not contain any other vertex. However, the satisfaction of the local proximity requirements in these types of drawings is often at the expenses of other important aesthetic constraints, such as having an uniform edge length and/or having an area of the representation that is polynomial with the size of the input graph (see, e.g. [10]). As a contrast, Euclidean Voronoi drawings only require a screen-area that is polynomial in the input size. For references on proximity drawings of graphs see [3].

The main results of this paper can be summarized as follows.

- We characterize Euclidean Voronoi drawable trees. Such trees are those whose interior vertices have degree at least three. Our result extends a previous result by Dillencourt [4] who proves that all maximal planar graphs are drawable as Delaunay triangulations, thus implying that any tree with internal vertices of degree exactly three is Euclidean Voronoi drawable.

- We present different algorithms that compute Euclidean Voronoi drawings of trees and that can satisfy different additional aesthetic constraints. We show how to compute radial Euclidean Voronoi drawings, i.e. Voronoi drawings of rooted trees in which the root of the tree is placed at the center of a disk and all vertices at distance $k$ from the root are drawn on a disk of radius $k$ and having center at the root. We also show how to compute uni-length Euclidean Voronoi drawings of trees, i.e. Euclidean Voronoi drawings where all edges have the same length. Further, we show that the above drawing can be computed so that all the the angles between the outgoing edges of each vertex in the drawing are constrained to be equal. The time complexity of the proposed algorithms is linear in the real RAM model of computation. 
- Motivated by the graphic appeal of drawing trees in a semi-orthogonal standard, we extend the investigation from the Euclidean to the Manhattan metric and study which trees are Manhattan Voronoi drawable. While the study of the Euclidean Voronoi drawable trees relies on a well-know theorem (see, e.g., [13]) which characterizes point sets whose Voronoi cells in the Euclidean metric are unbounded, as far as we know there is not a counterpart of such theorem in the Manhattan metric. We start our investigation of Manhattan drawings of trees by giving a characterization of the sets of points whose Voronoi diagrams in the Manhattan metric are trees.

- Since in the semi-orthogonal standard there cannot be more than eight edges incident on the same vertex there is a natural upper bound on the vertex degree of Manhattan Voronoi drawable graphs. Surprisingly, not only there cannot be Voronoi drawable trees whose vertices have degree eight, but we also show that there cannot exist drawable trees with vertices having degree larger than five. We also give examples of Manhattan drawable trees having vertices of degree five.

- We characterize those binary trees that are Manhattan Voronoi drawable. This result can be seen as the counterpart for the Manhattan metric of the result by Dillencourt [4] for the Euclidean metric.

For reasons of space, some proofs have been omitted or sketched in this extended abstract.

\section{Preliminaries}

Let $P=\left\{p_{0}, p_{1}, \ldots, p_{n-1}\right\}$ denote a set of $n$ distinct points in the plane. A point $p \in P$ will be called a site. For a given metric, we denote with $d(u, v)$ the distance function between two points $u$ and $v$.

Under the Euclidean or $L_{2}$ metric, the Voronoi region of a site $p_{i}$, denoted by $V\left(p_{i}\right)$ is the set of all points $q$ for which $d\left(q, p_{i}\right) \leq d\left(q, p_{j}\right)$ for all sites $p_{j}$, with $i \neq$ $j$. The set of all points $q$ for which $d\left(q, p_{i}\right)=d\left(q, p_{j}\right)$ is the Euclidean separator of $p_{i}$ and $p_{j}$ and is denoted as $\operatorname{Sep}\left(p_{i}, p_{j}\right)$. Under the Manhattan or $L_{1}$ metric, this definition is as follows [1]. Assume that $p_{i}$ is lexicographically smaller than $p_{j}$ and let $\operatorname{Dom}\left(p_{i}, p_{j}\right)=\left\{q \mid d\left(q, p_{i}\right)<d\left(q, p_{j}\right)\right\}$ and $\operatorname{Dom}\left(p_{j}, p_{i}\right)=\left\{q \mid d\left(q, p_{j}\right) \leq\right.$ $\left.d\left(q, p_{i}\right)\right\}$. The Manhattan separator of sites $p_{i}$ and $p_{j}$, denoted by $\operatorname{Sep}\left(p_{i}, p_{j}\right)$, is defined as the common boundary of $\operatorname{Dom}\left(p_{i}, p_{j}\right)$ and $\operatorname{Dom}\left(p_{j}, p_{i}\right)$. The Voronoi region of a site $p_{i}$ under the $L_{1}$ metric is $V\left(p_{i}\right)=\bigcap_{j \neq i} \operatorname{Dom}\left(p_{i}, p_{j}\right)$. Therefore the Voronoi region of a site in the $L_{1}$ metric in general does not contain all points of its boundary and may contain areas of thickness 0 . Since the characterization results and the drawing techniques of this paper are not affected by whether the Voronoi cells contain their boundaries or have areas of thickness 0, we assume throughout the paper that a Voronoi region of a site always contains its boundary both in the Euclidean and in the Manhattan metric, and does not contain areas of thickness 0 .

The Voronoi diagram of $P$, denoted by $\operatorname{Vor}(P)$ is the collection of all boundaries of the Voronoi regions $V\left(p_{i}\right)$. A point that lies in at least three different 
Voronoi regions is called a Voronoi vertex. Since vertices of a Voronoi diagram lie in at least three Voronoi regions, Voronoi drawable trees do not have vertices of degree 2.

Let $p$ be a point in the plane and let $l_{-}$and $l_{+}$be two lines of slope -1 and +1 through $p$ respectively. The lines $l_{-}$and $l_{+}$define four convex cones with apex $p$. We call East cone of $p$, denoted by $E C(p)$, the cone to the right of $p$, i.e. the cone that contains the positive $x-$ axis with $p$ as origin. Similarly, we define the North cone of $p(N C(p))$, West cone of $p(W C(p))$, and South Cone of $p$ $(S C(p))$ as the cones with apex $p$ and that are above, to the left, and below $p$ respectively. The cones $E C(p), N C(p), W C(p)$ and $S C(p)$ do not include points of $l_{-}$or $l_{+}$. The set of points of $l_{+}$with $x$ - and $y$-coordinates larger than those of $p$ is called the north-east bisector of $p$ and is denoted by ne $(p)$. The north-west bisector of $p$, denoted as $n w(p)$ is the portion of $l_{-}$whose points have larger $y$ and smaller $x$-coordinates than $p$ does. Similarly, the south-east bisector of $p$ (denoted as $s e(p)$ ), and the south-west bisector of $p$ (denoted as $s w(p)$ ) can be defined as the portions of $l_{+}$and $l_{-}$that lie in the third and fourth quadrant of $p$ and that do not contain $p$.

Let $C(p)$ be one of the four cones of $p$ defined above, i.e. $C=E C, N C$, $W C$ or $S C$. We use the notation $C[p]$ to denote the cone $C(p)$ including its boundary (but excluding the apex $p$ ). $C[p$ ) includes only the right boundary when the interior of the cone is viewed from $p$, and $C(p]$ is the cone plus the left boundary. For example, $E C[p]=E C(p) \cup s e(p) \cup n e(p), N C[p)=N C(p) \cup n e(p)$ and $N C(p]=N C(p) \cup n w(p)$. Finally we denote by $e(p)$ and $w(p)$ the points on the horizontal line through $p$ to the right and left of $p$ respectively, and by $n(p)$ and $s(p)$ the points on the vertical line through $p$ above and below $p$ respectively.

\section{$3 \quad$ Euclidean Voronoi Drawings of Trees}

In this section we first characterize the family of Euclidean Voronoi drawable trees and then show different algorithms that compute Euclidean Voronoi drawings of trees. Our characterization is based on the following result.

Theorem 1. [13] Let $P$ be a set of sites and let $p \in P$. The Voronoi region of $p$ is unbounded if and only if $p$ is a vertex of the convex hull of $P$.

By Theorem 1, the Voronoi diagram of $P$ is a tree if and only if all sites of $P$ are in convex position. In the next lemma we study sets of sites in convex position.

Lemma 1. Each tree without vertices of degree two is Euclidean Voronoi drawable.

Proof. Let $T$ be a tree without vertices of degree two. We prove the lemma by defining a set of sites $P$, for which $\operatorname{Vor}(P)$ is isomorphic to $T$. The edges of $\operatorname{Vor}(P)$ that have infinite length are replaced by edges of finite length at the end of the construction. We root $T$ at an arbitrary non-leaf vertex $r$. Suppose $r$ 
has degree $k$. Place a point $c_{0}$ on the plane at an arbitrary location and draw a circle $C_{0}$ around $c_{0}$ with radius 1 . Place $k$ sites on $C_{0}$ at arbitrary locations. The Voronoi diagram of these sites is a tree with a single non-leaf vertex of degree $k$ placed at $c_{0} ; c_{0}$ is the Voronoi vertex that represents $r$. For each site around $C_{0}$, draw a line tangent to $C_{0}$ through the site. We say that a point lies on the inside of one of these tangent lines if it lies on the same side as $c_{0}$. The remaining sites of $P$ that define the other internal Voronoi vertices of $T$ will all be placed outside the circle $C_{0}$ but inside the tangent lines.

We define the other points of $P$ such that $\operatorname{Vor}(P)$ is isomorphic to $T$ by traversing $T$ from $r$ to the leaves. The vertices at depth $m$ (i.e. the vertices whose path from $r$ consists of $m$ edges) are drawn only after all vertices at depth $m-1$ have been drawn. Suppose we want to draw vertex $v_{j}$ whose parent $v_{i}$ has been already drawn and let $c_{i}$ be the Voronoi vertex representing $v_{i}$. Vertex $v_{j}$ is drawn by defining a new disk whose center is on one of the infinite Voronoi edges incident on $c_{i}$. Let $e_{j}=\operatorname{Sep}(p, q)$ be this edge, where $p$ and $q$ are two sites already defined in a previous step of the algorithm. Let $C$ be the set of all circles whose centers define the Voronoi vertices drawn so far. Let $L$ be the set of all lines that are tangent to the circles in $C$ and that pass through the sites that have already been placed.

The following tasks are executed.

- Place vertex $c_{j}$ on the edge $e_{j}$, and draw the circle $C_{j}$ with center $c_{j}$ through $p$ and $q$. Place $c_{j}$ sufficiently close to $c_{i}$ so that $C_{j}$ is partially inside all tangent lines in $L$ and outside all circles in $C$.

- Let $k_{j}$ be the degree of vertex $v_{j}$ in $T$. Place $k_{j}-1$ sites on the portion of $C_{j}$ that lies inside all tangent lines in $L$ and outside all circles in $C$.

- Draw the $k_{j}-1$ lines through the new sites tangent to $C_{j}$. Add these lines to $L$ and add $C_{j}$ to $C$.

We can derive that the algorithm is correct based on the following two observations. Firstly, we can always place $c_{j}$ close enough to $c_{i}$ so that $C_{j}$ falls partially inside all tangent lines and outside all circles. Secondly the $k-1$ sites we place on $C_{j}$ are closer to $c_{j}$ than to any other vertex $c_{i}$, so the vertices of the Voronoi diagram of previously placed sites do not change.

The drawing algorithm defined in the proof of Lemma 1 can be modified in order to satisfy additional geometric constraints that define aesthetic criteria traditionally used in graph drawing to improve the readability of the drawings. For the definition of radial drawing and uni-length drawing see [2].

Theorem 2. Any tree $T$ whose internal vertices have at least three incident edges is Euclidean Voronoi drawable. A Euclidean Voronoi drawing $\Gamma$ of $T$ can be computed in time proportional to the size of $T$ in the real $R A M$ model of computation. $\Gamma$ can satisfy additional aesthetic requirements:

$-\Gamma$ can be a radial drawing, such that for each vertex $v \in \Gamma$ all outgoing angles of $v$ are the same.

$-\Gamma$ can be a uni-length drawing, such that for each vertex $v \in \Gamma$ all outgoing angles of $v$ are the same. 


\section{Point Sets in the Manhattan Metric}

We start our investigation of Manhattan Voronoi drawings of trees by giving a characterization of the sets of points whose Voronoi diagrams in the Manhattan metric are trees. This is achieved by independently characterizing sets of points whose Manhattan Voronoi diagrams are acyclic and those whose Manhattan Voronoi diagrams are connected.

Under the $L_{1}$ metric, a Manhattan separator $\operatorname{Sep}\left(p_{i}, p_{j}\right)$ is a semi-orthogonal polygonal chain, i.e. a chain whose segments can have slope $0, \infty$, and \pm 1 . An edge of a Manhattan Voronoi drawing is a subset of a Manhattan separator and hence it can have bends, each bend defining a turn of an angle that is a multiple of $\pi / 4$. The next property studies what a Manhattan separator can look like for a set of two sites.

Property 1. Let $p$ and $q$ be two sites. The eight possible shapes of the Manhattan separator $\operatorname{Sep}(p, q)$ are shown in figure 2 (a).
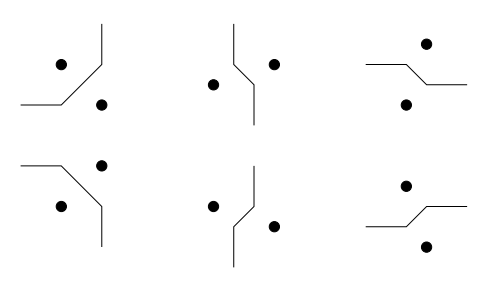

(a)

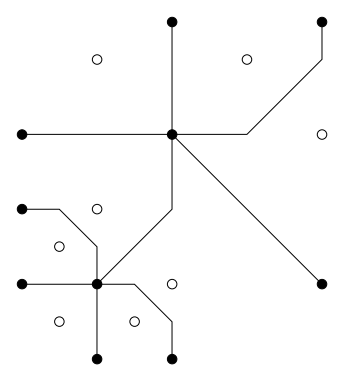

(b)

Fig. 2. (a) Manhattan separators of two sites. (b) Manhattan Voronoi drawing of a tree with two degree vertices of degree five.

We say that $p$ has the empty East cone property if $E C[p]$ does not contain sites. Similarly, $p$ has the empty North, empty West, and empty South cone property if $N C[p)$ does not contain sites, $W C[p)$ or $W C(p]$ does not contain sites, and $S C(p]$ does not contain sites respectively. We say that $p$ has an empty cone property if it has either the empty East, or North, or West, or South cone property. By exploiting Property 1 and the fact that the Voronoi region $V(p)$ of a site $p$ is a star-shaped polygon with $p$ in its kernel, the following can be proved.

Theorem 3. A Voronoi diagram $\operatorname{Vor}(P)$ is acyclic if and only if all sites $p$ have an empty cone property.

The Voronoi diagram of a set of sites in the Manhattan metric may be not connected. Intuitively, a Voronoi diagram is not connected when there exists a 
site such that two of its four cones are empty while the other two contain sites. However, since the cones may or may not contain their boundaries and since there can be sites aligned along lines with slope \pm 1 , a more precise statement of this condition is needed.

Lemma 2. If there is a site $p \in P$ such that $E C[p]$ does not contain sites, $N C(p]$ contains sites, $W C(p]$ or $W C[p)$ does not contain sites, and $S C[p)$ contains sites, then $\operatorname{Vor}(P)$ is not connected.

Lemma 3. If there is a site $p \in P$ such that $E C(p)$ contains sites, $N C[p)$ does not contain sites, $W C[p]$ contains sites, and $S C(p]$ does not contain sites, then $\operatorname{Vor}(P)$ is not connected.

Lemma 4. If there is a site $p \in P$ such that $E C(p] \cup N C[p)$ contains sites, $W C[p) \cup S C(p]$ does not contain sites, and $s w(p)$ contains sites, then $\operatorname{Vor}(P)$ is not connected.

Lemma 5. If there is a site $p \in P$ such that $S C(p] \cup E C[p)$ contains sites, $N C[p) \cup W C(p]$ does not contain sites, and $n w(p)$ contains sites, then $\operatorname{Vor}(P)$ is not connected.

A site $p$ is called a separating site if at least one of the sets of the conditions in the Lemmas 2, 3, 4, or 5 holds.

Theorem 4. Let $P$ be a set of sites and let $\operatorname{Vor}(P)$ be the Voronoi diagram of $P$ in the $L_{1}$ metric. $\operatorname{Vor}(P)$ is connected if and only if there exists no separating site $p \in P$.

Theorem 5. Let $P$ be a set of sites and let $\operatorname{Vor}(P)$ be the Voronoi diagram of $P$ in the $L_{1}$ metric. $\operatorname{Vor}(P)$ is a tree if and only if there is no separating site in $P$ and each site $p \in P$ has an empty cone property.

\section{Manhattan Voronoi Drawings of Trees}

Because of Property 1, the number of distinct edges incident on a same vertex of a Manhattan Voronoi drawing of a graph is at most eight. Hence, the class of Manhattan drawable trees is a contained in the class of those trees having maximum vertex degree at most eight. However, there is a smaller upper bound on the degree that the vertices of a Manhattan Voronoi drawable tree can have. Namely, by analyzing the geometry of the edges incident on a same Voronoi vertex it can be shown that there cannot be six or more edges incident on the same vertex. Based on Figure 2(b) that shows an example of a Manhattan Voronoi drawing of a tree with vertices of degree five, we can conclude the following.

Theorem 6. A Manhattan Voronoi drawable tree cannot have vertices with six or more incident edges. Also, there exist Manhattan Voronoi drawable trees that have vertices with five incident edges. 
Not all trees having vertices of degree at most five are Manhattan Voronoi drawable. To prove this claim, we characterize those binary trees that are Manhattan Voronoi drawable. We start by introducing some terminology.

Let $T$ be binary a tree without vertices of degree two. $T$ is a caterpillar if each vertex of $T$ is either a leaf or the neighbour of a leaf. Hence, a caterpillar contains a path that connects two leaves plus an arbitrary number of side branches consisting of single edges. The path is called backbone of the caterpillar; the branches are called legs of the caterpillar. Each branch shares a vertex with the backbone. If the caterpillar is a single edge, then it coincides with its backbone and there are no branches.

The class of binary trees that we are going to study consists of caterpillars "joined" to some "skeleton", which we define as follows. Let $T$ be binary tree without vertices of degree two. A spine of $T$ is a subtree of $T$ containing at least two and at most most four leaves of $T$ and containing all vertices and edges of the paths between these leaves. We say that a caterpillar is attached to a spine if one of the endpoints of its backbone is a non-leaf vertex of the spine.

Lemma 6. Any binary tree $T$ that has no vertices of degree 2 and that consists of an arbitrary number of caterpillars attached to a spine sp is Manhattan Voronoi drawable.

Proof. We prove the lemma assuming that that $s p$ has four leaves. If the spine $s p$ has fewer that 4 leaves, a similar reasoning can be used.

Notice that $s p$ has two vertices $v_{0}$ and $v_{1}$ of degree 3 whose neighbours are in $s p$. We explain how a set of sites $P$ can be constructed such that the Voronoi diagram of $P$ in the Manhattan metric is isomorphic to $T$.

We distinguish two different cases. Case (a): if there are no vertices between $v_{0}$ and $v_{1}$ then we start the construction as follows: sites $p_{0} p_{1}, p_{2}$ and $p_{3}$ are placed at points $(0,4),(6,0),(0,-4)$ and $(-6,0)$. Case $(b)$ : if there are vertices between $v_{0}$ and $v_{1}$ then we start the construction as follows: sites $p_{0} p_{1}, p_{2}, p_{3}$ and $p_{4}$ are placed at points $(-1,4),(1,4),(6,0),(0,-4)$ and $(-6,0)$.

We now add sites such that the Voronoi diagram of these sites includes the backbones of all caterpillars, but not yet the legs. This can be done by adding sites on the lines $y=4$ and $y=-4$ : place an arbitrary number of sites at points with $x$ - coordinates between -5 and -2 and between 2 and 5 . For case (b), we can also place sites on the line $y=4$ with $x$ - coordinates between -1 and 1 . It can be verified that the Voronoi vertices of the sites $p_{0}, p_{1}, p_{2}, p_{3}$ and $p_{4}$ do not move (except for the Voronoi vertex of $p_{0}, p_{1}$ and $p_{2}$ ), so the backbones of the caterpillars are attached to the correct sections of the spine.

Finally, we add legs to the backbones of the caterpillars as follows. Suppose that between sites $q_{0}$ and $q_{1}$ there is a caterpillar with $k$ legs. Assume that $q_{1}$ is to the right of $q_{0}$. Place $k$ sites on the ray $n w\left(q_{1}\right)$, close enough to $q_{1}$ to ensure that $N C\left[q_{0}\right)$ remains empty. Notice that the new sites do not change the location of any of the existing vertices in the Voronoi diagram of $P$, except the location of the vertex corresponding to $p_{0}, p_{1}$ and $p_{3}$ in case (b). It can be seen that the new sites create the legs as required. Therefore, all trees that can be decomposed into a spine and a set of caterpillars are Manhattan Voronoi drawable. 
Let $P$ be a set of sites whose Voronoi diagram $\operatorname{Vor}(P)$ is a tree in the Manhattan metric. The proof of the following lemma is based on an analysis of the properties of the cones defined by the sites in $P$.

Lemma 7. Any binary tree that is the Voronoi diagram of a set of sites under the $L_{1}$ metric is a tree $T$ that has no vertices of degree two and consists of an arbitrary number of caterpillars attached to a spine.

Lemmas 6 and 7 prove the following.

Theorem 7. A binary tree $T$ is Manhattan Voronoi drawable if and only if it has no vertices of degree 2 and consists of an arbitrary number of caterpillars attached to a spine.

\section{References}

1. F. Aurenhammer. Voronoi diagrams: A survey of a fundamental geometric data stru cture. ACM Comput. Surv., 23(3):345-405, Sept. 1991.

2. G. Di Battista, P. Eades, R. Tamassia, and I. G. Tollis. Graph Drawing. Prentice Hall, Upper Saddle River, NJ, 1999.

3. G. Di Battista, W. Lenhart, and G. Liotta. Proximity drawability: a survey. In R. Tamassia and I. G. Tollis, editors, Graph Drawing (Proc. GD '94), volume 894 of Lecture Notes Comput. Sci., pages 328-339. Springer-Verlag, 1995.

4. M. B. Dillencourt. Realizability of Delaunay triangulations. Inform. Process. Lett., 33(6):283-287, Feb. 1990.

5. M. B. Dillencourt. Toughness and Delaunay triangulations. Discrete Comput. Geom., 5:575-601, 1990.

6. M. B. Dillencourt and W. D. Smith. Graph-theoretical conditions for inscribability and Delaunayr ealizability. In Proc. 6th Canad. Conf. Comput. Geom., pages 287292, 1994.

7. P. Eades and S. Whitesides. The realization problem for Euclidean minimum spanning trees is NP-hard. Algorithmica, 16:60-82, 1996. (special issue on Graph Drawing, edited by G. Di Battista and R. Tamassia).

8. G. Liotta and G. Di Battista. Computing proximity drawings of trees in the 3dimensional space. In Proc. 4th Workshop Algorithms Data Struct., volume 955 of Lecture Notes Comput. Sci., pages 239-250. Springer-Verlag, 1995.

9. G. Liotta, A. Lubiw, H. Meijer, and S. H. Whitesides. The rectangle of influence drawability problem. Comput. Geom. Theory Appl., 10(1):1-22, 1998.

10. G. Liotta, R. Tamassia, I. G. Tollis, and P. Vocca. Area requirement of Gabriel drawings. In Algorithms and Complexity (Proc. CIAC' 97), volume 1203 of Lecture Notes Comput. Sci., pages 135-146. Springer-Verlag, 1997.

11. C. Monma and S. Suri. Transitions in geometric minimum spanning trees. Discrete Comput. Geom., 8:265-293, 1992.

12. A. Okabe, B. Boots, and K. Sugihara. Spatial Tessellations: Concepts and Applications of Voronoi Diagrams. John Wiley \& Sons, Chichester, UK, 1992.

13. F. P. Preparata and M. I. Shamos. Computational Geometry: An Introduction. Springer-Verlag, 3rd edition, 1988.

14. K. Sugihara and M. Iri. Construction of the Voronoi diagram for 'one million' generat ors in single-precision arithmetic. Proc. IEEE, 80(9):1471-1484, Sept. 1992. 